International Journal of Engineering \& Technology, $7(2.8)(2018) 550-553$
International Journal of Engineering \& Technology
SPC
Website: www.sciencepubco.com/index.php/IJET
Research Paper

\title{
Energy aware dynamic virtual machine consolidation in cloud data centers
}

\author{
G. Anusha, P. Supraja \\ Dept. of Information Technology, SRM University kattankulathur, India.
}

\begin{abstract}
Cloud computing is a growing technology now-a-days, which provides various resources to perform complex tasks. These complex tasks can be performed with the help of datacenters. Data centers helps the incoming tasks by providing various resources like CPU, storage, network, bandwidth and memory, which has resulted in the increase of the total number of datacenters in the world. These data centers consume large volume of energy for performing the operations and which leads to high operation costs. Resources are the key cause for the power consumption in data centers along with the air and cooling systems. Energy consumption in data centers is comparative to the resource usage. Excessive amount of energy consumption by datacenters falls out in large power bills. There is a necessity to increase the energy efficiency of such data centers. We have proposed an Energy aware dynamic virtual machine consolidation (EADVMC) model which focuses on pm selection, vm selection, vm placement phases, which results in the reduced energy consumption and the Quality of service (QoS) to a considerable level.
\end{abstract}

Keywords: Cloud computing, Resource utilization, VM Consolidation, VM Placement, energy consumption.

\section{Introduction}

Cloud Computing has been developing very rapidly in the recent years. It provides various resources to perform various tasks. It offers various benefits such as scalability, mobility, reliability, flexibility, affordability etc. These benefits have made it to take a well-founded place in the industry. Cloud Computing provides various services to its customers based on their requirements. It charges from the customers only for the amount of service they consumed i.e. pay -as-you-go basis. In the recent years because of cloud computing a huge number of data centers has been evolving. The most infrastructure needed for the cloud systems are made from the data centers which virtualizes thousands of high performance physical servers. This virtualization allows several virtual machines (VMs) to run simultaneously above a single physical machine (PM).The resources like CPU, Bandwidth, RAM and storage of a PM which are underlying are partitioned and shared by VMs present on it.

Modern data centers are the accommodating platforms to a extensive range of applications. These applications can run on several virtual machines positioned at different physical machines within a data center. The data centers consume large amounts of electrical energy for cooling, networking etc which results in high operational costs. Thus the energy consumption in the cloud data centers should be reduced, and this can be done by improving the way in which cloud resources are provisioned. Resource provisioning in cloud can be improved by using some of the ways like virtualization and live migration techniques. Many researchers focuses on the problem of energy efficient cloud computing through VM placement in data centers, by making sure that the resources are efficiently utilized to help the application workloads and to decrease the energy consumption. The main concern with the server virtualization is the VM Placement. It is the process of mapping VM requests onto the available physical machine resources based on some constraints or objective functions.

Generally the dynamic VM consolidation consists of two methods; migration of VMs from underutilized PM and then offloading VMs from PMs, when it suffers from overloading in order to avoid performance degradation or SLA violation. There were four major stages in VM consolidation scheme. They are under loaded PM detection, overloaded PM detection, VM selection, and VM placement. In this paper, an efficient upper and lower threshold value algorithm which helps in setting the upper and lower threshold values and Efficient Best Fit Decreasing Algorithm has been developed which allocates the VMs to the PM which is capable of providing the resources requested by the VMs and ensures optimum energy efficiency with improved QoS performance metrics.

The rest of this paper is structured as follows. In Section2 we discuss the related work and in Section 3 consists of the proposed work which consists of the system model and a description of the proposed approach, continuing with the experimental results in Section4. Finally we make a conclusion in Section5.

\section{Related Work}

Service providers want to decrease the overall operational cost incurred due to energy consumption. There are various solutions provided to minimize the energy consumption.

Thiago Kenji Okada et al. [19] presents the problem of energy efficient VM placement. They focuses on the placing a requested VM to a host. An effective VM placement aims to decrease the quantity of active physical machines by a way in growing the workload on the present active machines. To decrease the number of active PMs and also to suspend the PMs that are not in use, they 
proposed three algorithms which are the extension for the existing ones. Those are the First Fit Decreasing algorithm which was a modification of the greedy algorithm, the PABFD which will calculate the rise in power consumption once allocating of current $\mathrm{VM}$ to the host is done, storing the less power consumption and then VM is allocated to the host which has the least consumption and the idle physical hosts are suspended, the GPABFD algorithm in this the power consumption regarding the entire data center after simulation of the allocation of a VM to the current PM is done. A. Beloglazov et al.[2] presents a review of exploration in the energy efficient computing and they have proposed an algorithms for the VM selection and placement. The high level system architecture has been developed which consists of the mainly the four parts consumers/brokers, the green service allocator, VMs, Physical machines. For the VM placement phase the modified best fit decreasing algorithm has been proposed. For the VM selection they proposed three policies. They are minimization of migration policy- in which a minimum number of VMs are selected for migration from a PM in order to decrease the utilization of CPU below the upper threshold, The highest potential growth policy - migrates the VMs which has the lower usage of the CPU comparatively to the CPU size, The random choice policy - it selects the number of VMs randomly to reduce the CPU utilization by a PM. All the above three method are used when the CPU utilization is above the upper utilization threshold and the when the CPU utilization is below the lower utilization the VMs present on the PM are migrated to the other PM and the host is set to the sleep mode. Zhou et al. [21] proposed an adaptive three threshold energy area algorithms. It divides the data center hosts into four modules like the hosts with the little load, hosts with the light load, hosts with the moderate load and the hosts with the heavy load. The proposed method migrates the VMs from the heavily loaded or the little loaded host to the hosts that are lightly loaded, while the VMs present on the lightly and moderately loaded hosts will remain unchanged. The two types of adaptive three threshold algorithms has be done with the k-means average median and the interquartile method and for the vm selection the methods like minimum memory size policy and the lowest CPU utilization methods has been used.

Perla Ravi Theja et al. [14] presents adaptive genetic algorithm which is proposed for the VM consolidation which includes overload and under load utilization discovery, VM selection, and VM placement. For the host under load detection the PM which has the smallest utilization as matched with other host is found and whole VMs on that host are migrated to the other hosts and the PM is set to sleep mode. In the overloaded host the inter quartile range has been used to determine the upper threshold value. If and when the PM utilization is more than this upper threshold value then some of the VMs from that host should be migrated to other host. For the VM selection MMT policy has been used .It migrates VMs which require minimal time for migration than compared with the other VMs present on the PM. For the VM placement the adaptive genetic algorithm has been used which consists of the mapping, cross over the mutation and the placement. Ankita Choudhary et al. [3] proposed a method for optimizing the virtual machine placement using the bankers algorithm. The proposed method is named as the OVMPBA, which mainly focuses on determining the overloaded host using the dynamic threshold technique and minimizing migration policy for the VM selection. By using these two techniques an attempt has been made to efficiently utilize the resources and reduce the energy consumption. For the detecting the over loaded host the dynamic threshold technique has proposed. For the VM selection the MM policy is used, which selects the VM where the size of that was equivalent to difference between entire host utilization and threshold value. In [10] a random method is used in which the $\mathrm{pm}$ is selected randomly. The vm is placed on a randomly selected $\mathrm{PM}$ if the resources required by that VM are provided by the PM. Mohammed Rashid Chowdhury et al. [13] presents the various VM placement methods. The redesign of the various VM placement algorithms has been done and also they presented a technique in which clustering vms in order to migrate by considering into account the CPU utilization and the RAM capacity. The redesign of the worst fit decreasing algorithms has been done. The clustering technique has been proposed. The VMs in the dense cluster is now moved to the other hosts. The modified k-means algorithm has been used. It selects the optimal number of clusters by using the centroid method and selects the VMs to migrate and then uses the modified worst fit VM placement for the clustering i.e it the VMs from the cluster are allocated to a particular host.

\section{Proposed VM Consolidation Model}

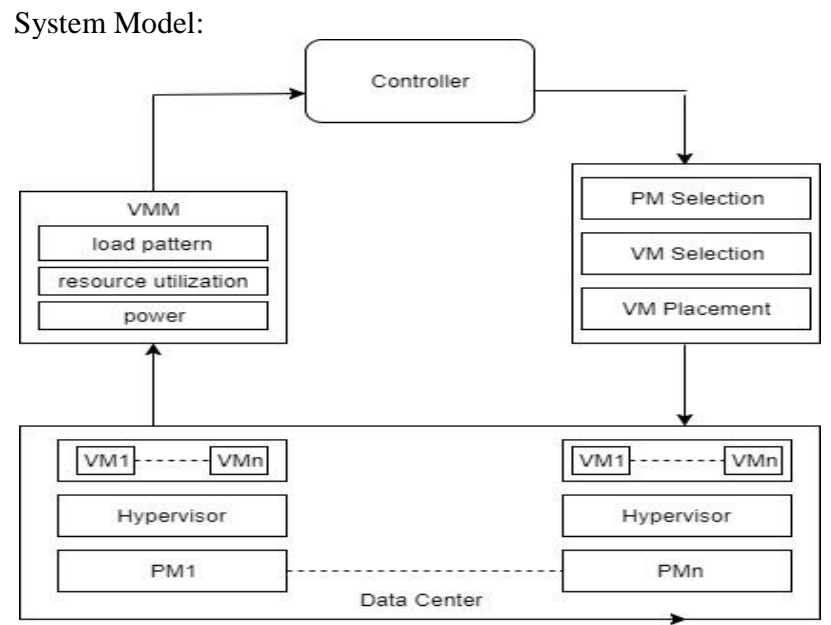

Fig. 1: Energy-aware Dynamic virtual machine Consolidation Model ( EADVMC)

The model contains a data center which consists of several PMs upon which a hypervisor is placed which helps the PM to create a number of VMs on it. Now the virtual machine monitor collects the data regarding the resource utilization, load pattern, power etc from the datacenter and sends the collected data to the controller. Based on the above collected data the controller checks whether the vm consolidation is required or not. Now if the consolidation is required the following stages, such as PM Selection, VM Selection, and VM Placement are performed.

\section{A. PM Selection:}

It consists of two phases such as the host overload detection and the host under load detection.

1) Host Overload Detection: When the VMs lying on the PM are more than the PM capacity then the PM gets overloaded. Due to this the energy consumption will increase. In order to reduce this, the overloaded PM has to be identified and that should be stabilized to its normal position. In order to identify the overloaded PM we propose an efficient upper threshold value algorithm which can be used to find out the upper threshold value according to the dynamically changing workloads. This threshold value can be used to find out the overloaded PM i.e if CPU utilization value of the PM is above the upper threshold value found from algorithm then that PM is overloaded. The algorithm includes the following steps:

Input: list of PMs

Output: upper threshold value

Step 1: sort the PMs in the list in the increasing order based on the utilization

Step 2: Now find the median of the PMs in the sorted list a) Now check for each PM in the list whose value is greater than the median value and obtain a new list of PMs .

b) Now consider a new PMs list obtained from (a).i.e the list of PMs greater than the median value

Step 3: Now find out the median of the new PMs list. 
Step 4: This median value obtained in step3 is the upper threshold value.

2) Host Under load Detection: If the CPU utilization of the $\mathrm{PM}$ is underutilized then the energy consumption may increase The PMs that are idle will consume more amount of energy. It is therefore likely to migrate all the VMs from underutilized PM to other PM ensuring that this migration is not overloading the new PM. For this to happen first the under loaded PM has to be selected. In order to do this we propose an efficient lower threshold value algorithm which helps to identify the under loaded PM. The algorithm includes the following steps:

Input: list of PMs

Output: lower threshold value

Step 1: sort the PMs in the list in the increasing order based on the utilization

Step 2: Now find the median of the PMs in the sorted list a) Now check for each PM in the list whose value is less than the median value and obtain a new list of PMs .

b) Now consider a new PMs list obtained from (a).i.e the list of PMs less than the median value

Step 3: Now find out the median of the new PMs list.

Step 4: This median value obtained in step3 is the lower threshold value.

\section{B. VM Selection:}

After selecting the PM from the above stage, now we need to select the VMs from the PM in order to migrate from it and bring

\section{Results}

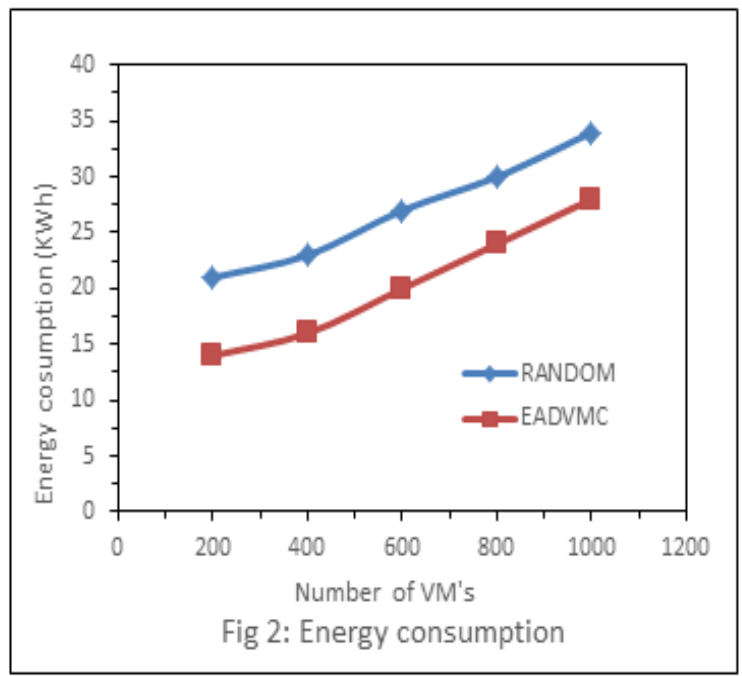

The simulations have been carried out in the cloudsim toolkit. The two graphs are shown which consists of the energy consumption and the VMs migration. We compare our proposed method with the random method. In fig2 the random method consumes high amount of energy and the EADVMC method consumes less energy. In fig3 the number of VMs migrated are less in EADVMC method than in random method. As the number of VMs migrated are less the QoS can be maintained.

\section{Conclusion}

In this paper we propose a EADVMC method. This helps in minimizing the energy consumption. This consists of four modules such as overload host detection, under load host detection, VM selection and VM placement which helps in determining the overloaded and under loaded PMs and then which VM has to be selected for migration followed by the VM placement on the appropriate pm. The proposed system helps in the PM to the normal state. For this all the VMs running on the under loaded PM has to be migrated and then the PM should be set to sleep/shutdown mode. In case of overloaded PM one or more VMs has to be selected for migration. The process of VM migration will consume energy and may even effect the QoS of the cloud applications. Therefore in order to reduce the number of VMs migration we have used the Minimum Migration Policy. In this policy the Euclidean distance is calculated between the load pattern of VM's and PM's. Note that the larger the distance, the effect of the PM is dominant.

\section{VM Placement:}

The VM placement is a major problem of the virtual resources addition. VM placement means finding out the best PM for the VM. The PM should be capable of providing the resources requested by the VM. The VMs that are selected in the above step should now be placed on the other PM by ensuring that the new PM which is accommodating these VMs should not get overloaded and the energy consumption should be less. For this we have proposed an efficient best fit decreasing algorithm which sorts the list of PMs in the increasing order of their cpu utilization and also the list of VMs are considered and now the VM searches for the appropriate PM by using the search method. The search method helps the VM to find out the best PM from the list.

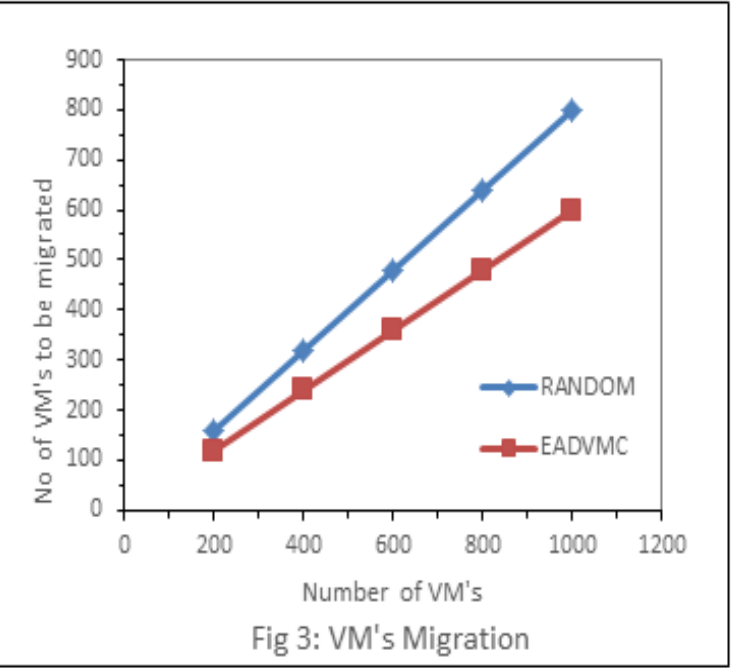

minimizing the energy consumption and also the SLA violation. As the number of VMs which has to be migrated are less hence the Qos can aslo be maintained. For evaluating the proposed work we used the cloudsim simulator. From the above results our proposed method results in reduced energy consumption.

\section{References}

[1] A. Beloglazov and R. Buyya, "Energy efficient resource management in virtualized cloud data centers," in 10th IEEE/ACM International conference on Cluster, Cloud Grid Computing, pp. 826-831, May 2010.

[2] A. Beloglazov, J. Abawajy, and R. Buyya, "Energy-aware resource allocation heuristics for efficient management of data centers for cloud computing," Future Generation Computer Systems, vol 28, pp. 755-768, May 2012.

[3] Ankita Choudhary, Dr.K.J.Mathai "Optimize Virtual Machine Placement in Banker Algorithm for Energy Efficient Cloud Computing" International Journal of Innovative Research in 
Computer and communication Engineering, vol 4, issue 4, April 2016.

[4] D. Frederico, C. Jose, F. Anderson, and G. Vinicius, "A systematic review on cloud computing," Journal of Supercomputing, vol. 68, pp. 1321-1346, June 2014.

[5] Dr. Naveen Kumar Gondhi and Mr. Paras Kailu "Prediction Based Energy Efficient Virtual Machine Consolidation in Cloud Computing" Second International Conference on Advances in Computing and Communication Engineering, 2015.

[6] Divya Bharathi P and Vamsee Krishna Kiran M "Virtual Machine Placement Strategies in Cloud Computing" International Conference on Innovations in Power and Advanced Computing Technologies [iPACT2017], 2017.

[7] E. Arianyan, H. Taheri , and S. Sharifian, "Novel Energy and SLA Efficient Resource Management Heuristics for Consolidation of Virtual Machines in Cloud Data Centers," Computers \& Electrical Engineering, vol. 47, pp. 222-240, 2015.

[8] Fahimeh Farahnakian, Tapio Pahikkala, Pasi Liljeberg, Juha Plosila and Hannu Tenhunen "Multi-Agent based Architecture for Dynamic VM consolidation in Cloud Data Centers" 40 th IEEE Euromicro Conference on Software Engineering and Advanced Applications, 2014.

[9] G. Keller, M. Tighe, H. Lutfiyya, and M. Bauer, "An analysis of first fit heuristics for the virtual machine relocation problem,', in Proc. 8th International Conference Workshop System Virtualization Management (SVM) Network Service Management. (CNSM), pp. 406-413, Oct. 2012.

[10] Harshit Verma, Surat Kumar Dhal "Energy Effficient Virtua Machine Migration in Cloud data centers" dissertion, National Institute of Technology, Rourkela,2015.

[11] Jiankang Dong, Xing Jin, Hongbo Wang, Yangyang Li, Peng Zhang, Shiduan Cheng "Energy-Saving Virtual Machine Placement in Cloud Data Centers" 13 th IEEE/ACM International Symposium on Cluster, Cloud, and Grid Computing, 2013.

[12] Li Hongyou1, Wang Jiangyong, Peng Jian, Wang Junfeng, Liu Tang, "Energy-Aware Scheduling Scheme Using Workload Aware Consolidation Technique in Cloud Data Centres," China Communications, pp.114-124. Dec 2013.

[13] Mohammed Rashid Chowdhury, Mohammad Raihan Mahmud, Rashedur M.Rahman "Implementation and performance analysis of various VM placement strategies in CloudSim" Journal of Cloud Computing, vol 4, issue 1, Dec 2015.

[14] Perla Ravi Theja, S.K.Khadar Babu "An Evolutionary Computing based Energy Efficient VM Consolidation Scheme for Optimal Resource Utilization and QoS Assurance" Indian journal of Science and Technology, vol 8, issue 26, Oct 2015

[15] Qi Chen, Jianxin Chen, Baoyu Zheng, Jingwu Cui and Yi Qian "Utilization-based VM Consolidation Scheme For Powe Efficiency in Cloud Data Centers" IEEE ICC-Cloud Computing Systems, Networks, and Applications(CCSNA), 2015.

[16] R.N.Calheiros, R.Ranjan, A.Beloglazov, C.A.F.DeRose, and R.Buyya, "CloudSim: A toolkit for modeling and simulation of cloud computing environments and evaluation of resource provisioning algorithms,' Softw. Pract. Exper., vol. 41, pp. 23 50, Sep. 2011.

[17] R.Ranjana,S.Radha and J.Raja :Performance study of resource aware energy efficient VM Placement Algorithm" IEEE WiSPNET Conference, 2016.

[18] Saad Mustafa, Kashif Bilal, Sajjad A.Madani, Samee U.Khan, Nikos Tziritas and Laurence T.Yang "Performance Evaluation of Energy-aware Best Fit Decreasing Algorithms for Cloud Environments" IEEE International Conference on Data Science and Data Intensive Systems, 2015

[19] Thiago Kenji Okada, Albert De La Fuente Vigliotti "Consolidation of VMs to Improve Energy Efficiency in Cloud Computing Environments" Computer networks and distributed systems, 2015.

[20] Y. Lee and A. Zomaya, "Energy efficient utilization of resources in cloud computing systems," Journal of Supercomputing, vol. 60, pp. 268-280, May 2012.

[21] Zhou, Zhigang Hu, and Keqin Li. "Virtual machine placement algorithm for both energy-awareness and sla violation reduction in cloud data centers." Scientific Programming, 2016. 